\title{
Digital Archaeological Archiving in Sweden: the Swedish National Data Service perspective
}

Ulf Jakobsson

\section{Summary}

In the past, data from archaeological investigations, as well as research projects led by universities have not been archived or made publicly accessible. Synthetic publications such as papers, reports and articles have been available, but not the underlying data files containing the original data to be reused or combined with new/other datasets for further research.

Archaeological investigations are regulated within Sweden, but it has only recently been possible for that data to be preserved and disseminated in a more streamlined way. The mandatory requirement to archive research data at universities is often not enforced, resulting in a loss of data that is very problematic. This is now slowly changing owing to requirements from both governmental bodies and funding agencies, and therefore the future of archaeological data in Sweden looks a bit brighter.

\section{Introduction}

Within Sweden each public authority (including higher education institutions and museums) is responsible for the archiving of data created within that organisation. This responsibility can be transferred via agreements (ratified by the Swedish National Archive) or an organisation can be designated by the government to archive the material created by other organisations. For many years, however, the mandatory archiving of research data, including archaeological and historical data, was not enforced within Swedish universities. There are a variety of reasons for this, but the primary issue was a lack of appropriate systems for handling research data, leading to loss of irreplaceable information.

There are several legal frameworks related to archaeology and cultural heritage in Sweden. Cultural heritage and the cultural environment are protected by a number of 
different legal frameworks, laws and directives. Most important of these is the Historic Environment Act. However, for research data created within universities, the Public Access to Information and Secrecy Act [PDF] must also be followed. This article will present how data produced/created within universities are now being handled, as part of the mission of the national research infrastructure, the Swedish National Data Service (SND). It will also present the ongoing work by universities in cooperation with SND, to create a national infrastructure where research data can be described, stored and made openly available.

\section{Research Data in Universities}

Research publications are most often submitted to journals or other outlets based on academic merit. Digital and/or analogue information, documentation or data, created as a product of university research may be indexed and archived within that university, but this has not been, and is not (yet) always the case. Research data created within universities often ended up in a box on a bookshelf, despite university and researcher requirements to archive and share the data. In most cases, this results from a lack of appropriate archival systems for digital research data; the researcher has no place to deposit and archive their data. In addition, university researchers have typically considered such data to be their private property, to be used only for their own publications.

Archaeological data produced or collected within universities are of a myriad of file formats and sizes; born-digital as well as digitised. Most of the data is produced as part of a research project but also, for example, during student training excavations. For most projects, the researcher applies for funding and then collects or creates the data during the project, writes their articles and reports, and the project data either lives on their local hard drive, or within a networked personal folder within the university cloud storage (not an archive!). It has never been mandatory to archive and share data produced within a project, not even data derived from a non-repeatable archaeological excavation, meaning such data are lost to future researchers.

\subsection{Research excavations and regulations}

If a researcher or group of researchers at a university wish to conduct a research excavation, they must obtain permission from one of the 21 local County Administrative Boards (CAB) (one for each county). These CAB are state authorities with regional responsibility for cultural heritage management, and thereby control the permissions to perform archaeological excavations within Sweden. Rules and regulations regarding excavations are written by the Swedish National Heritage Board (NHB), to which the $\mathrm{CAB}$, the University, and others who conduct archaeological excavations must adhere.

The NHB originated in 1630 and is one of Sweden's oldest government agencies under the auspices of the Ministry of Culture. It is the central administrative agency in the area of cultural heritage and cultural or historic environment. This includes (among others), community planning and construction, grant distribution, and monitoring and supporting regional cultural heritage management.

The existing legal frameworks, laws and directives are important for the NHB's activities and for cultural heritage work both within and outside the country's borders. The majority 
of decisions, in relation to the Historic Environment Act, concerning the regional and local level are made by the 21 County Administrative Boards. For digital resources within the National Heritage Board, see Löwenborg et al., this issue.

\subsection{Archaeological fieldwork}

Archaeological fieldwork in Sweden is in most cases undertaken as a form of mitigation prior to development projects - new roads, buildings, railroads etc., but in only a few cases as research projects. In each case, a permit must be obtained from the local CAB. In short, there are three levels of investigations:

1. archaeological enquiry (arkeologisk utredning);

2. archaeological preliminary investigation (arkeologisk förundersökning);

3. archaeological survey/excavation (arkeologisk undersökning/utgrävning).

Investigations are always conducted in this order, but not every investigation requires all three levels.

Archaeological enquiries are the lowest level of investigation and their purpose is to determine the presence or absence of any ancient/historical remains within an area subject to development.

An Archaeological preliminary investigation is undertaken if ancient/historical remains are suspected, or found to be present, prior to excavation. It can also be used to find and determine the borders of a site so that it might be possible to relocate a planned development. These preliminary investigations are more extensive and are made to give the $C A B$ sufficient basis for deciding if an archaeological survey/excavation is required, including removal of all or part of the site. If finds are discovered during the preliminary investigation, they are deposited with the appropriate museum.

Archaeological surveys/excavations are undertaken to document and understand a site in accordance with scientific methods and traditions, and are most often conducted when it is not possible to avoid affecting the site during development. Usually, an archaeological survey means that the site is excavated, resulting in an investigation that cannot be repeated. Finds from the excavation are sent to the appropriate museum, but the general public and researchers are also informed through articles, exhibitions and lectures.

The reports, including information about possible finds, the area of investigation, maps etc. must be sent to the NHB and the local CAB, when the investigation is finalised.

\section{The Swedish Research Council and Open Access to Research Data}

In 2013, the Swedish Research Council (Vetenskapsrådet, VR) was commissioned by the government to produce national guidelines for Open Access to scientific information, which resulted in the report Proposal for national guidelines for Open Access to scientific information in 2015. In 2016, VR was commissioned to coordinate national work on 
introducing Open Access to research data. This conversion is to be implemented no later than 2026. At the same time the National Library of Sweden was tasked with the corresponding assignment for publications. In 2017, the VR was commissioned to develop criteria for assessing how research data meet the FAIR principles, presented in the report Kriterier för FAIR forskningsdata (Swedish only).

National funding agencies like Formas, Forte, Riksbankens jubileumsfond, and of course VR, require Open Access to the research data more and more. Taken together, these efforts are creating change in how research data is handled in Sweden, at least when it comes to archiving and publicly sharing the data.

\section{Swedish National Data Service (SND)}

The Swedish National Data Service (SND) is a national research infrastructure mandated by VR, and certified with the CoreTrustSeal. SND, with its predecessor SSD (Swedish Social Science Data Service), has been curating, storing, disseminating, and making research data available for more than 40 years. Over the last ten years, this has included archaeological data. SND has an online data catalogue where the deposited data are described and can be freely and directly downloaded, or made accessible on request and by order (where access to data is limited due to restrictions, for example because the data include personal details or other sensitive information). In these cases the principal researcher may conduct a confidentiality assessment before the data can be released. There are also several online resources regarding data management, legal questions, file formats, etc. It has never been mandatory to deposit data with SND (or any other place) so the inflow of archaeological data has not exceeded capacity.

\subsection{SND and the past}

As mentioned previously, there are no formal requirements for researchers to deposit data with SND, which means all SND data are deposited by choice. Until 2020, when a researcher wished to disseminate their data, they sent a copy of the data and its documentation to SND. SND then reviewed the data, the metadata, and the documentation provided to ensure it all met deposit requirements. Such requirements included sufficient documentation/metadata so that a secondary user could find and assess the information, and determine the usefulness of the data, but also whether the data are in a common format, that it is well documented and preferably non-proprietary. If everything was in order, a metadata landing page catalogue post was produced and published (e.g. https://snd.gu.se/en/catalogue/study/snd2004). A DOI was also provided for the dataset, ensuring the data could be found and cited. The requirements and archiving processes are in accordance with the OAIS-model for data preservation and the FAIR Principles established as best practice for facilitating data use. SND is not a formal data archive from a Swedish legal perspective, but still handles data as if it were. This included the conversion of file formats better suited for archiving, with the data stored and distributed over several secured servers. 


\subsection{SND and the present}

Until 2018, the Social Sciences, the Humanities, and Health Medicine were the main data depositors to SND. From 2018 onwards SND was given a remit to cover all research areas. To accomplish this, SND became a consortium of nine universities. SND also initiated a network of so-called Data Access Units (DAU) for each university. These units typically consist of librarians, legal advisors, archivists, and IT staff. However, it is up to the university to decide the composition and number involved. Today there are approximately 30 higher education institutions and public research institutes in the network, but the number is growing. These organisations are required to safely store and archive their research data, while also making the data publicly available in accordance with the Open Access ethos 'as open as possible, as closed as necessary'. The idea is that data are no longer going to be deposited to SND (except in exceptional cases) but safely stored at each institution, while still described and accessed in one portal via SND's metadata catalogue. This satisfies their archival obligations, the Open Access guidance from the EU, and the requirements the Swedish Research Council has been assigned to implement by the Swedish government.

Researchers can log into SND's management system (called DORIS), fill in a deposit form (which includes a choice of several metadata/subject profiles), and 'upload' their data. If the researcher's university has a functional DAU with their own dedicated storage area, the data are sent to the DAU and the DAU can start processing the metadata and the data files. If the university does not have a storage area the data are sent to SND (as long as no sensitive information is included). As before, this must include sufficient, good-quality metadata and documentation. Data files must be of good quality, preferably in non-proprietary formats, etc. It is possible to publish data elsewhere and still have metadata published in SND's catalogue. The requirements for this are similar, with the exception that a persistent identifier (typically a DOI) must be provided by the depositor.

Currently, the major problem is that not all universities have a functional DAU with staff skilled in data management, the ability to adhere to the FAIR Principles, or have completed the administrative and strategic work necessary to implement a DAU. In addition, very few universities have created the data-storage protocols and/or infrastructure to archive their research data. Therefore, SND together with SUNET (Swedish University Computer Network) and SNIC (The Swedish National Infrastructure for Computing) are creating a possible permanent storage solution for the universities. This storage area, at SUNET or SNIC, will only be accessible by the institutions. SUNET is a research infrastructure that meets the need for data communication at Swedish universities and other public organisations in connection with research or higher education. SNIC is a national research infrastructure that makes available large-scale high performance computing resources, storage capacity, and advanced user support, for Swedish researchers. SND will continue to organise training workshops, meetings, and courses to ensure each DAU receives the appropriate support so they may implement the archiving and dissemination processes previously carried out by SND. 


\subsection{SND and the future}

The current vision of SND is for each university to have their own dedicated storage area, either locally or via SUNET/SNIC. Each university should also have a fully functional DAU that can support their researchers regarding data management, legal questions and storage, etc. Researchers should be able to publish data produced within research projects in accordance with the FAIR principles and Open Access, either via SND's metadata catalogue or elsewhere, in accordance with best practice for their domain. The aim of SND is to become more of a knowledge and service centre, while still maintaining a certified repository for those researchers who cannot make their data available via a university.

\subsection{Research data catalogue and archaeology/history data}

By the end of 2020, data deposited with SND from the disciplines of archaeology and history included around 470 archaeological investigations (GIS-databases and corresponding Access databases), around 40 databases (primarily in Excel and Filemaker Pro), along with vector files in Adobe Illustrator. All but ten of these are freely available for direct download without registration. The data are also made visible via the ARIADNEplus portal.

Most of the data deposited in GIS format were originally created using proprietary software called Intrasis. In the past, this presented a major problem, as it is used for most Swedish investigations, and cannot be exported to other file formats. However, through the work of the project URDAR (see Löwenborg et al., this issue for further information) this will hopefully be solved soon. That said, a research group and some students led by Daniel Löwenborg at the University of Uppsala managed to convert Intrasis data into shapefiles and Access databases, and were able to deposit it with SND.

SND does not have any qualitative information about who has used the data, as by law we are not permitted to ask for this information, or require user registration; nor do we know how much has actually been reused. We do have quantitative data, which shows a general increase in access each year, with a large jump in 2019 , although this could be attributed either to a change in the methodology, or to connections from the ARIADNE portal. 
Table 1: Number of datasets directly downloaded and 'tagged' with Archaeology and/or History 2015-2020

2015

3046

2016

2609

2017

1047

2018

2129

2019

14203

2020

14008

\section{Conclusion}

Archaeological research data, created both within an academic research project or prior to development, has not traditionally been archived or made publicly available. Due to an increased awareness that research data is a resource that should be more available to the public and to new research projects, better infrastructures and guidelines are now continuously under development in Sweden. In addition, requirements from governmental bodies and funding agencies are more and more outspoken in advocating for these issues and resources.

The demand for Open Access to research data has started a frenetic development within Swedish universities to solve problems associated with both long-term archiving and the need to make data publicly available. This, however, is a massive aim/project/goal that will take years to achieve, and will still only cover newly produced data. The backlog of legacy data is another problem that hopefully also will be solved in the future. Firstly, however, laws, directives, guidelines, technical facilities to store research data, as well as administrative systems to describe and share research data must be implemented. Researchers at higher education institutions, museums and commercial archaeologists must become better at documenting their data in a FAIR, streamlined and standardised way. Still, the future for archaeological data in Sweden continues to look brighter.

Historic Environment Act https://www.raa.se/in-english/cultural-heritage/historicenvironment-laws/historic-environment-act-1988950/ [Last accessed: 8 January 2021].

Kriterier för FAIR forskningsdata, https://www.vr.se/analys/rapporter/vararapporter/2018-12-07-kriterier-for-fair-forskningsdata.html [Last accessed: 12 January 2021].

Public Access to Information and Secrecy Act, English translation of the brochure 'Offentlighetsprincipen och sekretess - kortfattat om lagstiftningen', which briefly 
describes the Public Access to Information and Secrecy Act (SFS

2009:400). https://www.regeringen.se/4a76f3/contentassets/2c767a1ae4e8469fbfd0fc04 4998ab78/public-access-to-information-and-secrecy.pdf [Last accessed: 12 January 2021].

Proposal for national guidelines for Open Access to scientific information. https://www.vr.se/english/analysis/reports/our-reports/2015-03-02-proposalfor-national-guidelines-for-open-access-to-scientific-information.html [Last accessed: 12 January 2021]. 